\title{
What's the difference?
}

- on the textual effects of language campaigns in public institutions

\section{ANNE KJÆRGÅRD}

During the last 30-40 years, the Danish public sector has seen a substantial amount of language campaigns, i.e. campaigns aimed at changing the traditional way of addressing citizens in writing. However, research evaluating the effects of such campaigns is scarce

The paper has two main purposes: First of all it presents the results of an analysis examining the textual effects of language campaigns in the Municipality of Copenhagen and the Courts of Denmark. Drawing on Linell's concept of recontextualisation, a comparative analysis of texts written by four selected individuals before and after the language campaigns is presented. Secondly the text-analytical method is presented and discussed. The text analysis draws on the corpus linguistic tool Word Smith Tools and includes 3 analytical approaches: a top down approach, an approach elaborating the results of the top down approach and an explorative approach.

The analysis shows that the analysed texts in the two organisations have not undergone substantial changes. However, the analytical approach outlined in the article does provide the analyst with a comprehensive overview of the textual changes that may (or may not) occur as a result of a language campaign. 